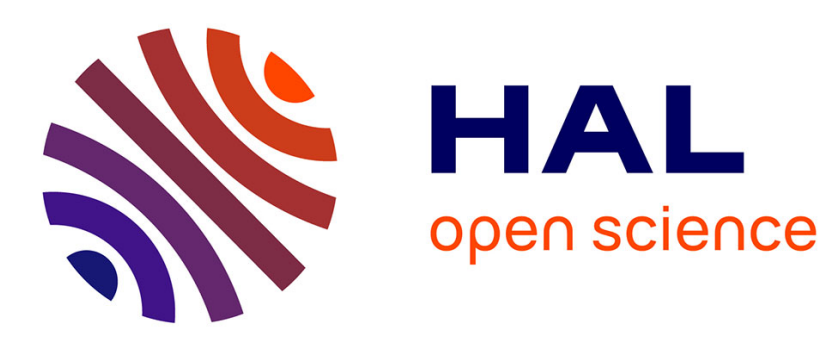

\title{
Constitutive modeling of the anisotropic behavior of Mullins softened filled rubbers
}

\author{
Yannick Merckel, Julie Diani, Mathias Brieu, Julien Caillard
}

\section{To cite this version:}

Yannick Merckel, Julie Diani, Mathias Brieu, Julien Caillard. Constitutive modeling of the anisotropic behavior of Mullins softened filled rubbers. Mechanics of Materials, 2012, 57, pp.30-41. 10.1016/j.mechmat.2012.10.010 . hal-00992343

HAL Id: hal-00992343

https://hal.science/hal-00992343

Submitted on 16 May 2014

HAL is a multi-disciplinary open access archive for the deposit and dissemination of scientific research documents, whether they are published or not. The documents may come from teaching and research institutions in France or abroad, or from public or private research centers.
L'archive ouverte pluridisciplinaire HAL, est destinée au dépôt et à la diffusion de documents scientifiques de niveau recherche, publiés ou non, émanant des établissements d'enseignement et de recherche français ou étrangers, des laboratoires publics ou privés. 


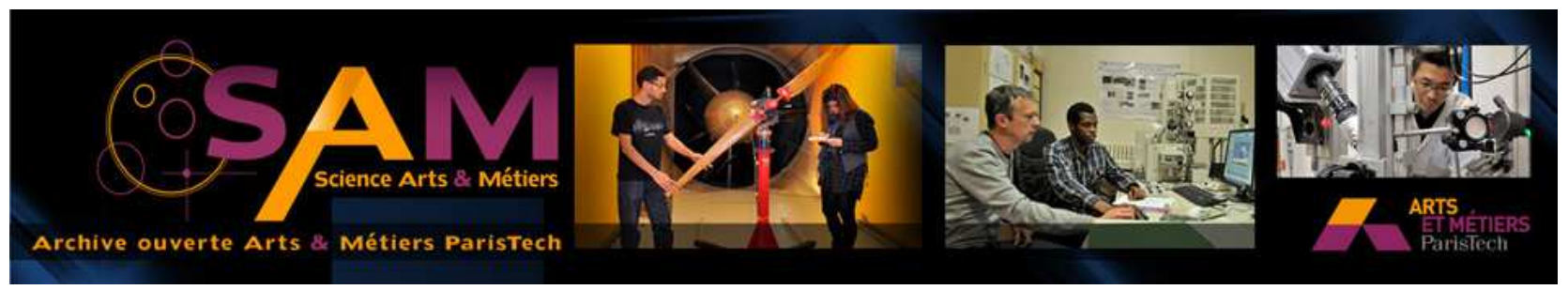

Science Arts \& Métiers (SAM)

is an open access repository that collects the work of Arts et Métiers ParisTech researchers and makes it freely available over the web where possible.

This is an author-deposited version published in: http://sam.ensam.eu

Handle ID: .http://hdl.handle.net/10985/8156

\section{To cite this version :}

Yannick MERCKEL, Julie DIANI, Mathias BRIEU, Julien CAILLARD - Constitutive modeling of the anisotropic behavior of Mullins softened lled rubbers - Mechanics of Materials - Vol. 57, p.30-41 2012 


\title{
Constitutive modeling of the anisotropic behavior of Mullins softened filled rubbers
}

\author{
Yannick Merckel $^{\mathrm{a}}$, Julie Diani ${ }^{\mathrm{b}, *}$, Mathias Brieu $^{\mathrm{a}}$, Julien Caillard ${ }^{\mathrm{c}}$ \\ ${ }^{a}$ LML, CNRS, Ecole Centrale de Lille, bd Paul Langevin, 59650 Villeneuve d'Ascq, France \\ b Laboratoire PIMM, CNRS, Arts et Métiers ParisTech, 151 bd de l'Hôpital, 75013 Paris, France \\ ${ }^{\mathrm{c}}$ Manufacture Française des Pneumatiques Michelin, CERL, Ladoux, 63040 Clermont-Ferrand, France
}

Keywords:

Filled rubber

Mullins softening

Anisotropy

Constitutive modeling

Non-proportional loading

\begin{abstract}
A B S T R A C T
Original constitutive modeling is proposed for filled rubber materials in order to capture the anisotropic softened behavior induced by general non-proportional pre-loading histories. The hyperelastic framework is grounded on a thorough analysis of cyclic experimental data. The strain energy density is based on a directional approach. The model leans on the strain amplification factor concept applied over material directions according to the Mullins softening evolution. In order to provide a model versatile that applies for a wide range of materials, the proposed framework does not require to postulate the mathematical forms of the elementary directional strain energy density and of the Mullins softening evolution rule. A computational procedure is defined to build both functions incrementally from experimental data obtained during cyclic uniaxial tensile tests. Successful comparisons between the model and the experiments demonstrate the model abilities. Moreover, the model is shown to accurately predict the non-proportional uniaxial stress-stretch responses for uniaxially and biaxially pre-stretched samples. Finally, the model is efficiently tested on several materials and proves to provide a quantitative estimate of the anisotropy induced by the Mullins softening for a wide range of filled rubbers.
\end{abstract}

\section{Introduction}

Filled rubbers undergo substantial stress softening and possible residual stretch when first loaded. This phenomenon, first reported by Bouasse and Carrière (1903), was studied intensely by Mullins (1947, 1949, 1950, 1969) and is now commonly referred to as the Mullins softening. By performing successive non-proportional loadings (i.e. successive loadings with changing the directions of stretching or the type of loading), Mullins (1947, 1949) was first to point out softening induced anisotropy. However, subsequent experimental studies mainly focused on proportional loadings and the induced anisotropy was

\footnotetext{
* Corresponding author. Tel.: +33 1442461 92; fax: +33 144246290 E-mail addresses: yannick.merckel@gmail.com (Y. Merckel), julie. diani@ensam.eu (J. Diani), mathias.brieu@ec-lille.fr (M. Brieu), julien. caillard@fr.michelin.com (J. Caillard).
}

not investigated further for several decades. Only recently, several studies (Laraba-Abbes et al., 2003; Hanson et al., 2005; Diani et al., 2006; Itskov et al., 2006; Dargazany and Itskov, 2009; Machado, 2011; Merckel et al., 2012) brought to light Mullins softening induced anisotropy by application of successive non-proportional loadings.

In terms of modeling, one may find a significant number of models in the literature designed to reproduce the behavior of Mullins softened rubber-like materials. However, most of these models are developed for idealized isotropic softening and very few aim at capturing the softening induced anisotropy. A first representation for anisotropic hyperelastic behavior was proposed by Weiss et al. (1979), based on strain invariants which limits its applicability to simple anisotropies (transverse isotropy or orthotropy) and excludes its extension to the Mullins softening. An alternative approach based on directional behavior laws was proposed by Pawelski (2001), Göktepe 
and Miehe (2005) and Diani et al. (2006). The directional laws were shown to capture the Mullins softening induced anisotropy without major difficulties by considering that damage evolves independently along each material direction. Nonetheless, in the existing directional laws, the residual stretch is constrained by the Mullins softening induced anisotropy, and this is not in complete agreement with the experimental observations. Actually, the residual stretch is very dependent of the material viscoelasticity as shown by the substantial and rapid recovery after unloading (Mullins, 1949; Diani et al., 2006), whereas the Mullins softening is commonly considered as irreversible at room temperature. Additional experimental observations detailed in the following section support a decoupling of the residual stretch with the Mullins softening. Therefore, both should be accounted for independently.

The pre-cited directional models are based on a physical interpretation of the Mullins softening. They generally depend on physically motivated elementary strain energy densities and the Mullins softening is accounted for by altering the strain energy density parameters. In order to accurately fit original experimental data, the elementary strain energy density and the Mullins softening evolution rule may require substantial modifications according to the material behavior. Moreover, the strain energy density and the evolution rule must be guessed a priori, and no general procedure has been proposed to do so.

In this study, our main motivation is to propose a general framework versatile for the modeling of hyperelastic rubber-like material behavior with a realistic account of the anisotropic induced Mullins softening. For this purpose, a directional approach is considered with an anisotropic criterion for the Mullins softening activation. At first, according to experimental evidences, Mullins softening and residual stretch evolutions are decoupled. Then, in order to propose a model with the largest flexibility, the account for the Mullins softening is chosen to avoid assumptions on the elementary strain energy density or the softening evolution rule. This is made possible by using the strain amplification concept proposed by Mullins and Tobin (1957). Finally, an identification procedure is proposed to assess both the elementary strain energy density and the Mullins softening evolution rule without postulating their mathematical forms.

The paper is organized as follows. In the next section, the experimental setup and experimental results are presented. The constitutive equations and the identification procedure are detailed in Section 3, and results are shown and discussed in Section 4. Finally, concluding remarks close the paper.

\section{Experiments}

\subsection{Experimental setup}

In this study, we used carbon-black filled styrene butadiene rubbers (SBR) prepared by Michelin. Materials varied according to their filler amounts from 30 to 60 phr. Details of the rubber compositions are listed in Table 1. All materials were manufactured into $2.5 \mathrm{~mm}$-thick sheet shape.
Table 1

Material compositions in parts per hundred rubber (phr).

\begin{tabular}{llllll}
\hline Ingredient & A & B1 & B2 & B3 & B4 \\
\hline SBR & 100 & 100 & 100 & 100 & 100 \\
Carbon-black (N347) & 40 & 30 & 40 & 50 & 60 \\
Antioxidant (6PPD) & 1.0 & 1.9 & 1.9 & 1.9 & 1.9 \\
Stearic acid & - & 2.0 & 2.0 & 2.0 & 2.0 \\
Zinc oxide & - & 2.5 & 2.5 & 2.5 & 2.5 \\
Structol ZEH & 3.0 & - & - & - & - \\
Accelerator (CBS) & 1.5 & 1.6 & 1.6 & 1.6 & 1.6 \\
Sulfur & 1.5 & 1.6 & 1.6 & 1.6 & 1.6 \\
\hline
\end{tabular}

In-plane isotropy was verified by testing in uniaxial tension, samples punched in various directions. However, due to the manufacturing process consisting in rubber mixing within a two-roll mill and in pressure molding, full isotropy is unlikely and we will discuss this aspect in Section 2.4. The material labeled A (Table 1 ) is used as a reference material to illustrate experimental grounds of the model and to validate the model and the identification procedure. Materials B1 to B4 will be used to assess the general aspect of the model and to test its interest for comparing the mechanical behavior of various materials.

Mechanical tests were conducted on two devices. Uniaxial loadings were performed on an Instron 5882 testing machine. Biaxial loadings were applied by an in-lab built planar biaxial testing machine controlled by four perpendicular electromechanical actuators. All tests were run at a constant crosshead speed chosen in order to reach an average strain rate close to $10^{-2} \mathrm{~s}^{-1}$ in the maximum stretched direction. In order to genuinely characterize the Mullins softening, virgin material samples of $30 \mathrm{~mm}$ long and $4 \mathrm{~mm}$ wide normalized dumbbell shape were submitted to cyclic uniaxial tension tests with increasing maximum stretch at each cycle. Then, in order to study the Mullins induced softening, some samples were uniaxially or biaxially pre-stretched. Uniaxial pre-loadings were applied on large dumbbell specimens $25 \mathrm{~mm}$ wide and $60 \mathrm{~mm}$ long, while biaxial pre-loadings were conducted on cross shape samples. Small dumbbell samples $4 \mathrm{~mm}$ wide and $10 \mathrm{~mm}$ long were punched in the pre-stretched samples and submitted to cyclic uniaxial tensions with increasing maximum stretch.

During loadings, local stretches were measured by video extensometry using four paint marks on the free faces characterizing the in-plane principal stretches. In the case of uniaxial tension tests, all three principal stretches may be measured using two cameras, each one facing one of the sample free faces. In what follows, the states of stretch are characterized by the principal stretches which coincide with the eigenvalues $F_{i i}$ of the deformation gradient $\boldsymbol{F}$. The direction of larger stretching will be referenced as direction 1, directions 2 and 3 are perpendicular to direction 1 and direction 3 lies along the sample thickness. For uniaxial loadings, $\lambda$ may conveniently denote the principal stretch in the tension direction. The Cauchy stress $\sigma_{11}=F / S$ is used for uniaxial tension responses, with $F$ the force and $S$ the current sample cross-section. Let us note that incompressibility was generally assumed when computing the Cauchy stress. This assumption will be discussed in the next section. 


\subsection{Material incompressibility}

In order to reach the uniaxial Cauchy stress, the current sample cross-section $S$ has to be measured while stretching the sample. The relation between the current section $S$ and the initial section $S_{0}$ is given by $S=F_{22} F_{33} S_{0}$. In order to lighten the experimental setup, incompressibility (leading to $S=\lambda^{-1} S_{0}$ ) is conventionally assumed. Nevertheless, substantial volume changes have been reported within stretched non-crystallizing filled rubbers (see references within Le Cam, 2010). Therefore, volume changes upon stretching was investigated in material $A$ by recording the three principal stretches.

Fig. 1a shows the volume changes occurring during a monotonic loading and during a cyclic loading with an increasing maximum stretch of $\Delta \lambda=1$ at each cycle. One observes that the material volume does not increase significantly as long as the material stretching remains below $\lambda \approx 3$.5. The existence of such a stretching threshold has already been reported by Shinomura and Takahashi (1970) and Zhang et al. (2012). Fig. 1 also shows that under monotonic tension volume expands with the stretching while under cyclic loading conditions, no significant volume changes occur as long as the stretching remains below the maximum stretch previously applied. Similar observations have been obtained by dilatometry measurements by Mullins and Tobin (1958). Therefore, while assuming incompressibility seems unrealistic upon the first stretch, it shows to be a fair assumption for subsequent stretchings below the maximum stretch ever applied (Fig. 1b). Since this study focuses on Mullins softened behavior, the unloading Cauchy stress-strain response may be computed using the incompressibility assumption. The model will be proposed within an incompressible framework.

\subsection{Mullins softening and residual stretch}

When a filled rubber is submitted to cyclic loading conditions, one may notice along with the softening, a residual stretch that increases with the applied maximum stretch. Both features are usually pointed out as consequences of the Mullins effect. However, some experimental evidences show otherwise. The softening occurring upon first stretch is an irreversible damage phenomenon at room tempera- ture (Mullins, 1947). To the contrary, the residual stretch is very dependent of viscoelasticity and shows an important and rapid recovery at room temperature (Mullins, 1949; Diani et al., 2006). Other experimental observations prove that although residual stretch and material softening usually occur simultaneously, their evolutions are not necessarily correlated. Various loading histories with identical maximum stretch may result in substantial residual stretch changes while the Mullins softening remains unaffected. An example is presented in Fig. 2. A $\lambda=2.5$ uniaxialy pre-stretched sample is submitted to uniaxial a cyclic loading with an increasing maximum stretch of $\Delta \lambda=0.25$ at each cycle after a $72 \mathrm{~h}$ stress free recovery. Fig. 2a shows the loading responses resulting from the cyclic loading. One may notice that the stress-stretch responses evolve at each cycle from the very first cycle. However, while representing the loading stress-stretch responses applying a residual stretch correction, according to $\lambda_{\text {cor }}=\lambda / \lambda_{\text {res }}$, one notices that the loading responses superimpose well until the maximum previous stretch $(\lambda=2.5)$ is reached (Fig. 2b). Then material behavior changes due to the Mullins softening occurrence are observed for subsequent cycles. This demonstrates that in Fig. 2a, the mechanical behavior of the material does not evolve for cycles below $\lambda=2.5$, except for the residual stretches, evidencing a significant residual stretch evolution and a constant Mullins softening.

Other experimental evidences support the uncorrelation of the residual stretch and of the Mullins softening. First, the Mullins softening and the residual stretch seem both quite dependent to the material composition but with different sensitivities (Mullins, 1949; Dorfmann and Ogden, 2004; Merckel et al., 2011). Second, some materials may evidence some large Mullins softening with very little residual stretch. For instance, a silicone filled rubber studied by Machado (2011) displays significant Mullins softening without noticeable residual stretch.

\subsection{Anisotropy characterization}

When submitting a sample to a uniaxial tension according to direction 1 , its free faces are submitted to the boundary conditions $\sigma_{22}=\sigma_{33}=0$, and when the measured stretches satisfy to $F_{22}=F_{33}$, the material shows transverse (a)

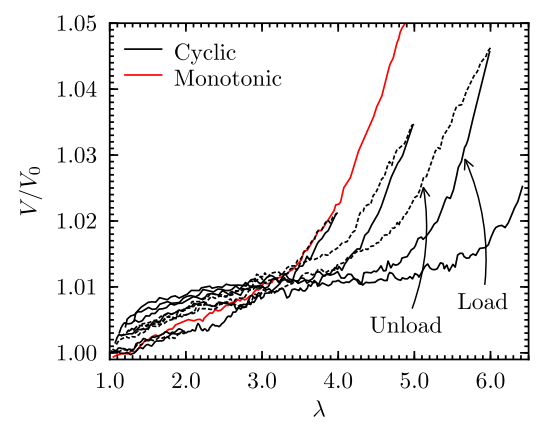

(b)

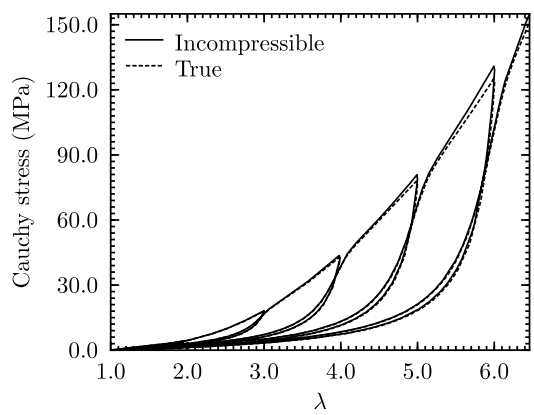

Fig. 1. (a) Material A volume changes while submitted to monotonic and cyclic uniaxial tension. (b) Effect of material A incompressibility loss on its cyclic uniaxial tension stress-stretch response. 
(a)

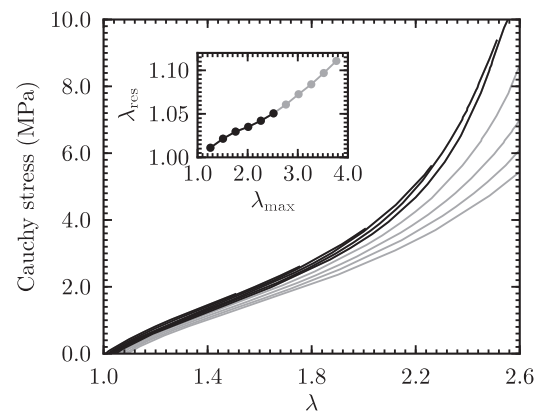

(b)

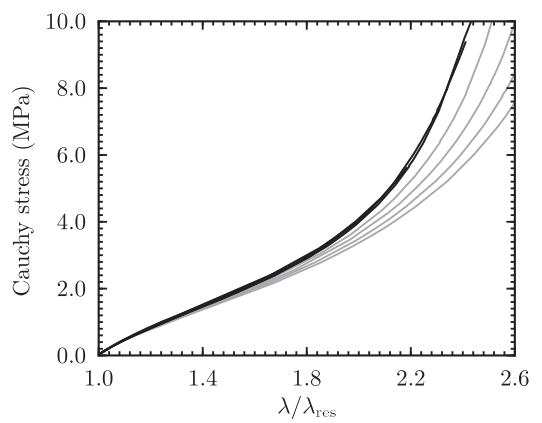

Fig. 2. Uniaxial tensile cyclic test performed after a $72 \mathrm{~h}$ stress-free recovery on a 2.5 -uniaxialy pre-stretched material. (a) Stress-stretch $(\lambda, \sigma)$ loading responses. (b) Residual stretch corrected stress-stretch $\left(\lambda / \lambda_{\text {res }}, \sigma\right)$ loading responses.

isotropy properties. Therefore in order to illustrate the material anisotropy, the ratio $F_{22} / F_{33}$ resulting from a cyclic proportional uniaxial tension test (loading shown in Fig. 1) is plotted with respect to the stretch $\lambda$ in Fig. 3a. The ratio $F_{22} / F_{33}$ appears different from 1 , highlighting the material initial anisotropy resulting from the manufacturing process. The material appears stiffer along the plate thickness direction than in any in-plane direction (keep in mind that the in-plane isotropy has been verified). More interestingly, the $F_{22} / F_{33}$ evolution seems to follow the same path for every cycle, evidencing the same anisotropy throughout the test.

The anisotropy characterization method is now applied to a small uniaxial dumbbell sample punched in a $\lambda=2.5$ equi-biaxially pre-stretched specimen. Results are shown in Fig. 3b. The $F_{22} / F_{33}$ evolution follows the same path as long as the sample is stretched below the maximum stretch previously applied $(\lambda=2.5)$. Then the anisotropy evolves at each cycle and the ratio $F_{22} / F_{33}$ slowly evolves toward a similar path than the path displayed by the virgin material in Fig. 3a. The introduced material anisotropy characterization will provide an additional element to validate the relevance of the modeling.

\subsection{Equilibrium response}

In the current paper, the material viscoelasticity is not considered and our focus is set on the equilibrium

(a)

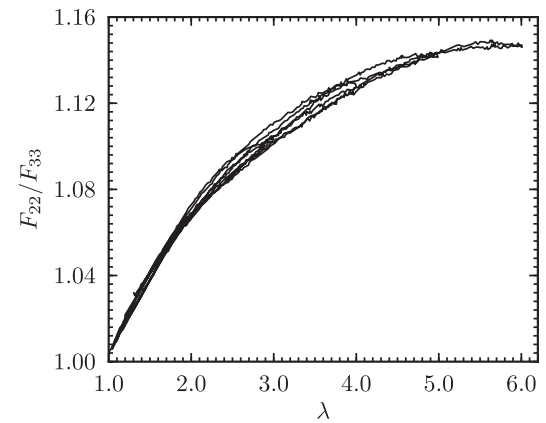

responses only. Once the Mullins softening has been evacuated, the loading and the unloading responses are fairly close and both responses may be used to characterize the material softened behavior (Fig. 4a). In order to remain consistent with previous modeling works proposed by the authors (Diani et al., 2006), the unloading responses are favored, and the material mechanical behavior evolution due to the Mullins softening is illustrated by the stress-stretch responses in Fig. 4b.

The next section presents the theoretical and the computational aspects of the modeling.

\section{Modeling}

\subsection{Hyperelastic framework}

Experimental observations reported in Section 2.3 support a decoupling of the residual stretch from the Mullins softening. Therefore, we use a kinematic approach decomposing the total deformation gradient $\boldsymbol{F}$ into an elastic part $\boldsymbol{F}_{e}$ and an inelastic part $\boldsymbol{F}_{p}$,

$\boldsymbol{F}=\boldsymbol{F}_{e} \boldsymbol{F}_{p}$

The inelastic deformation gradient tensor $\boldsymbol{F}_{p}$ changes the initial reference configuration into a stress-free intermediate configuration accounting for the residual deformation, while the elastic deformation gradient tensor $\boldsymbol{F}_{e}$ changes the stress-free configuration into the current configuration and therefore accounts for the elastic deformation. It is

\section{(b)}

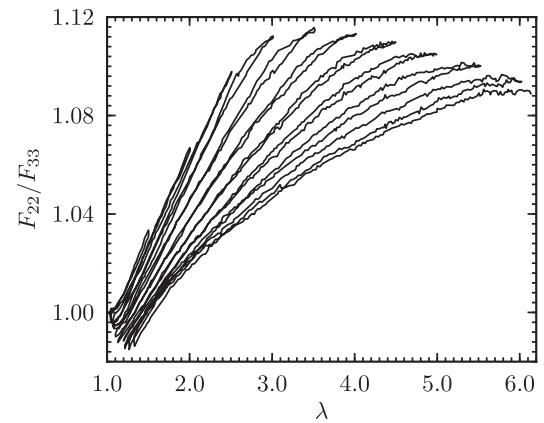

Fig. 3. In-plane (2,3) anisotropy changes. (a) Proportional cyclic uniaxial tension loading. (b) Cyclic uniaxial tension loading performed on a 2.5-biaxially pre-stretched sample. 
(a)

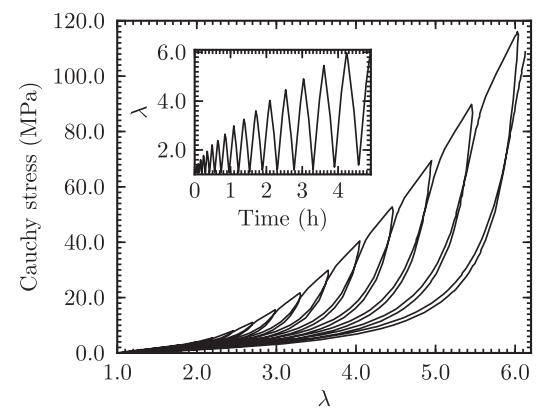

(b)

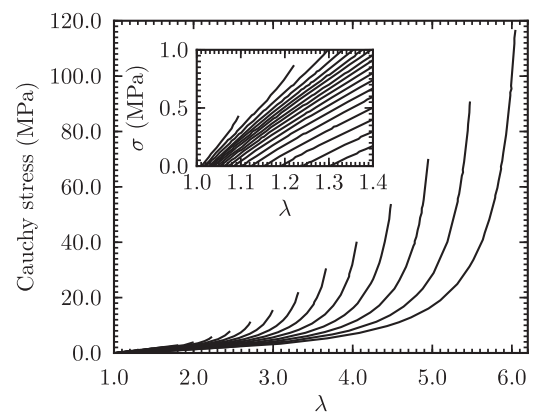

Fig. 4. Material stress-stretch response to a uniaxial tensile cyclic test with maximum stretch increasing at each cycle. (a) Entire response. (b) Unloading responses.

assumed that $\boldsymbol{F}_{p}$ does not evolve during the unloading responses shown in Fig. $4 \mathrm{~b}$.

In order to describe the material deformation, the right Cauchy-Green tensor $\boldsymbol{C}=\boldsymbol{F}^{t} \boldsymbol{F}$ and the left Cauchy-Green tensor $\boldsymbol{F}=\boldsymbol{F}^{t} \boldsymbol{F}$ are introduced (superscript $t$ denotes transposition). The state of the material is assumed to be described by the strain energy $\mathcal{W}$ written in terms of $\boldsymbol{C}_{e}$ and $\boldsymbol{B}_{p}$. Considering a strain energy with decoupled effects of the elastic and inelastic deformations leads to,

$\mathcal{W}\left(\boldsymbol{C}_{e}, \boldsymbol{B}_{p}\right)=\mathcal{W}_{e}\left(\boldsymbol{C}_{e}\right)+\mathcal{W}_{p}\left(\boldsymbol{B}_{p}\right)$

and the second Piola-Kirchhoff stress tensor in the stressfree configuration derives from the second law of thermodynamics,

$\boldsymbol{S}=2 \frac{\partial \mathcal{W}_{e}\left(\boldsymbol{C}_{e}\right)}{\partial \boldsymbol{C}_{e}}$

Elastomeric materials can be represented as threedimensional networks of very long flexible macromolecules randomly oriented in all directions of space. In directional approaches, the strain energy density $\mathcal{W}_{e}$ is evaluated from the summation of elementary strain energy contributions $w$ over all considered directions. An idealized representation introduced by Treloar and Riding (1979) is the fullnetwork model, which considers a continuous spatial distribution of directions leading to an integration over the unit sphere,

$\mathcal{W}_{e}\left(\boldsymbol{C}_{e}\right)=\iint_{\mathcal{S}} w(\boldsymbol{u}) \mathrm{d} \mathcal{S}$

with unit vectors $\boldsymbol{u}=(\cos (\theta), \sin (\theta) \cos (\varphi), \sin (\theta) \sin (\varphi))$ characterized by the polar angles $(\theta, \varphi)$ and $\mathrm{d} \mathcal{S}=1 /(4 \pi) \sin (\theta) \mathrm{d} \varphi \mathrm{d} \theta$.

In such a directional representation, anisotropy may be accounted for by considering uneven elementary strain energy contributions $w$, according to the direction $\boldsymbol{u}$. As previously noticed by Diani et al. $(2004,2006)$ and Göktepe and Miehe (2005), such an account for anisotropy may lead to uncontrolled residual stresses in the free-strain state. Therefore, in order to circumvent undesired residual stresses, and to satisfy to a stress-free undeformed state, the constitutive equation, Eq. (3), is modified into (Diani et al., 2004),
$\boldsymbol{S}=2 \frac{\partial \mathcal{W}_{e}\left(\boldsymbol{C}_{e}\right)}{\partial \boldsymbol{C}_{e}}-\left.2 \frac{\partial \mathcal{W}_{e}\left(\boldsymbol{C}_{e}\right)}{\partial \boldsymbol{C}_{e}}\right|_{\boldsymbol{c}_{e}=\boldsymbol{I}}$

The elastic extension along each direction $\boldsymbol{u}, \Lambda_{e}$, is obtained from the right elastic Cauchy-Green tensor as,

$\Lambda_{e}=\sqrt{\boldsymbol{u} \cdot \boldsymbol{C}_{e} \cdot \boldsymbol{u}}$

Let us note that $\partial \Lambda_{e} / \partial \boldsymbol{C}_{e}=(\boldsymbol{u} \otimes \boldsymbol{u}) / 2 \Lambda_{e}$, hence the elastic energy density partial derivative comes as

$\frac{\partial \mathcal{W}_{e}}{\partial \boldsymbol{C}_{e}}=\frac{1}{2} \iint_{\mathcal{S}} \frac{\boldsymbol{u} \otimes \boldsymbol{u}}{\Lambda_{e}} \frac{\partial w}{\partial \Lambda_{e}} \mathrm{~d} \mathcal{S}$

The Cauchy stress tensor $\boldsymbol{\sigma}$ is obtained by pushing forward the Piola-Kirchhoff stress tensor $\boldsymbol{S}$ from the relaxed configuration to the current configuration via $\boldsymbol{F}_{e}$. Substituting Eq. (7) in Eq. (5) and assuming material incompressibility yield to the following expression for the Cauchy stress tensor,

$\boldsymbol{\sigma}=\boldsymbol{F}_{e}\left(\iint_{\mathcal{S}} g(\boldsymbol{u})(\boldsymbol{u} \otimes \boldsymbol{u}) \mathrm{d} \mathcal{S}\right) \boldsymbol{F}_{e}^{t}-p \boldsymbol{I}$

where $p$ is an arbitrary hydrostatic pressure introduced to account for incompressibility and $g(\boldsymbol{u})$ a directional scalar that writes,

$g=\frac{f\left(\Lambda_{e}\right)}{\Lambda_{e}}-f(1)$

with $f$ the elementary force-extension relation in the direction $\boldsymbol{u}$ defined as

$f\left(\Lambda_{e}\right)=\frac{\partial w\left(\Lambda_{e}\right)}{\partial \Lambda_{e}}$

While the full-network model initially proposed by Treloar and Riding (1979) uses a specific inverse Langevin function based form for $f$, the above formulation is not restricted and can be applied to any directional force-extension $f\left(\Lambda_{e}\right)$. Therefore, we do not assume any specific mathematical form for $f$ since the latter will unfold upon experimental data fit.

The full-network framework is not efficient for numerical implementations due to the numerical integrations and in order to circumvent the time-consuming computational integration task, discrete integrations are usually 
preferred. For this purpose, a finite number of directions is considered. For instance, Göktepe and Miehe (2005) and Diani et al. (2006) used sets of 42 and 32 directions respectively, based on Bazănt and Oh (1986) numerical integrations. We followed this path but many other methods may be found in the literature.

In the next section, account for the Mullins softening is introduced.

\subsection{Mullins softening}

Recently, Merckel et al. (2012) conducted an extensive experimental study on the Mullins softening. It was shown that the latter evolves when at least one material direction is stretched above its maximum stretch. Therefore, the criterion proposed by Diani et al. (2006) for anisotropic Mullins softening has been validated by Merckel et al. (2012) experimental work. It writes as,

$\exists \boldsymbol{u}(\theta, \varphi) \mid\left(\Lambda-\Lambda_{\max }\right)=0$

with $\Lambda$ being the total extension along direction $\boldsymbol{u}$

$\Lambda(\boldsymbol{u})=\sqrt{\boldsymbol{u} \cdot \boldsymbol{C} \cdot \boldsymbol{u}}$

and $\Lambda_{\max }$ the maximum of $\Lambda$ over the loading history

$\Lambda_{\max }(\boldsymbol{u})=\max _{\tau \in[0 ; t]}[\Lambda(\boldsymbol{u}, \tau)]$

with $t$ the current time. It is noteworthy that the criterion Eq. (11) is based on the total extension and not only on its elastic component. This particular aspect of the criterion is supported by the fact that a softened material may recover some of its residual stretch without recovering any of its Mullins softening as shown in Section 2.3. Once the criterion defined, the damage variable which provides the softening in the stress-strain responses remains to be introduced.

The strain amplification concept, early introduced by Mullins and Tobin (1957), and based on experimental evidences reproduced by Klüppel and Schramm (2000) and Merckel et al. (2011) for instance, supports the idea that the stress-strain responses of softened filled rubbers evolve due to the amplification of the strain undergone by softened materials compared to virgin materials at similar stress. This may be written as,

$\log \left(\Lambda_{e}\right)=X \log \left(\Lambda_{e}^{\text {virgin }}\right)$

when the logarithmic strain is chosen. The strain amplification factor $X$ satisfies to $X(\boldsymbol{u}) \geqslant 1$ along each direction $\boldsymbol{u}$. The stretches $\Lambda_{e}^{\mathrm{virgin}}$ and $\Lambda_{e}$ characterize the directional stretch in the virgin material and the directional amplified stretch respectively. The strain amplification factor concept is introduced within the hyperelastic framework proposed in Section 3.1 by substituting $\Lambda_{e}$ as a function of $X$ and $\Lambda_{e}^{\text {virgin }}$ (Eq. (14)), in $g$ (Eq. (9)). Such a concept was already used in a similar fashion by Qi and Boyce (2004).

At this point, the model is fully defined. The residual stretches are captured by $\boldsymbol{F}_{p}$ in the decomposition Eq. (1). The Cauchy stress response, for a general full-network directional framework, is given by Eq. (8). The Mullins criterion is a directional criterion, defined by Eq. (11), providing possible induced anisotropy. Finally, the Mullins softening is accounted for by substituting Eq. (14) in $g$ (Eq. (9)), the enhanced directional stretches $\Lambda_{e}$ depending on the directional amplification factors $X(\boldsymbol{u})$. In order to describe and predict the material softening depending on the loading history, two material functions remain to be determined: $X$ and $f$. Therefore the next section draws attention to the identification procedure.

\subsection{Identification of the elementary force-extension function $f$ and the Mullins softening rule $X$}

The elementary force-extension function and the Mullins softening evolution rule are built incrementally in order to obtain a good fit of the experimental unloading responses from a cyclic uniaxial tension test with increasing maximum stretch at every cycle as shown in Fig. 4. Since for every mechanical test, the material stress-stretch response depends on both $f$ and $X$, an original method is defined guaranteeing simultaneous identification of both functions.

The main difficulty stands in the uneven evolution of the softening according to the spatial directions. Actually, the maximum directional extension $\Lambda_{\max }(\boldsymbol{u})$ depends on the direction considered ranging from 1 to $\lambda_{\max }$ (the maximum stretch in the uniaxial stretching direction). Fig. 5 illustrates $\Lambda_{\max }$ directional evolution for uniaxial tension tests.

When unloading the sample, the Mullins softening and the residual stretch evolutions are both stabilized. The inelastic part $\boldsymbol{F}_{p}$ (see Eq. (1)) coincides with the residual stretch $\lambda_{\text {res }}$ and the elastic part $\lambda_{e}$ may be extracted from the measured stretch $\lambda^{\text {meas }}$ using,

$\lambda_{e}=\frac{\lambda^{\text {meas }}}{\lambda_{\text {res }}}$

The material anisotropy evolves upon stretching only. Therefore, when the incompressible material is assumed as initially isotropic, the relation $F_{22}=F_{33}=\lambda^{-0.5}$ is obtained for uniaxial stretching in direction 1 . Constitutive equations Eq. (8), simplify into:

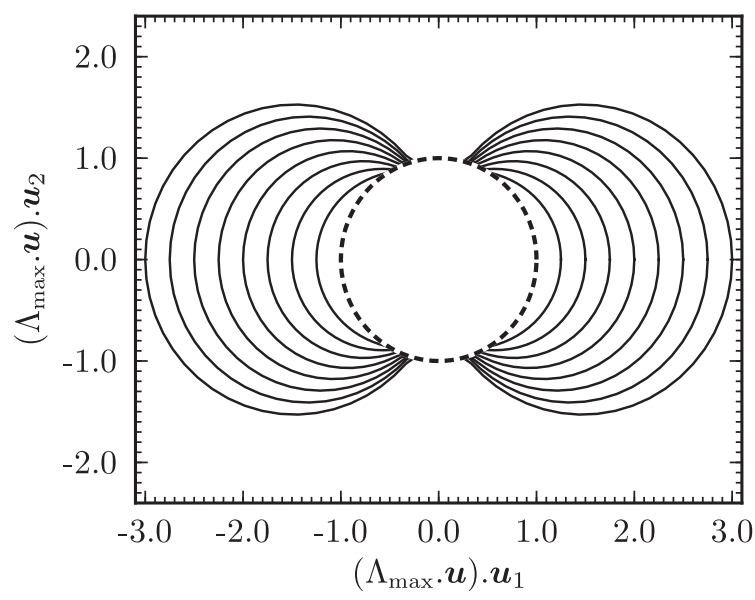

Fig. 5. Directional evolution of $\Lambda_{\max }$ during a cyclic uniaxial loading, projected in the sample plane $\left(\boldsymbol{u}_{1}, \boldsymbol{u}_{2}\right)$. The initial state is emphasized by dashed line. 
$\sigma_{11}=\iint_{\mathcal{S}} g\left(u_{1}^{2} \lambda_{e}^{2}-\frac{u_{2}^{2}}{\lambda_{e}}\right) \mathrm{d} \mathcal{S}$

with $g(\boldsymbol{u})$ depending on $\Lambda_{e}(\boldsymbol{u}), X(\boldsymbol{u})$ and $f$ (Eqs. (14) and (9)).

On one hand $X$ increases with the increase of the maximum stretch submitted and remains constant during unloadings. On the other hand, $f$ is independent of the softening, and remains the same for any cycle. The identification strategy is based on a resolution of the implicit Eq. (16). From a given experimental couple $\left(\sigma_{11}, \lambda_{e}\right)$ and knowing the Mullins softening governing parameter spatial density $\Lambda_{\max }(\boldsymbol{u})$ illustrated in Fig. 5, local values $X$ and $f$ may be computed numerically. Therefore, discrete definitions for $f$ and $X$ may be built by putting into practice the procedure synopsized in Fig. 6.

The identification procedure starts from the first (and smallest) cycle. Initial conditions $X(1)=1$ and $f(1)=0$ are naturally chosen, then $X$ and $f$ are progressively extended. In the first cycle $(i=1)$, the identification process is initialized according to the procedure reported in Table 2 in order to compute $X$ for $\Lambda_{\max } \in\left[1, \lambda_{\max }^{(i=1)}\right]$ and $f$ for $\lambda_{e} \in\left[1, \lambda_{e \max }^{(i=1)}\right]$. It is worth noting that a small cycle is advised for the initialization.

Once $X$ and $f$ have been initialized, they are extended by fitting each unloading response as illustrated in Fig. 6. At cycle $(i)$, the beginning of functions $X$ and $f$ have previously been determined, and the next identification action is performed in two steps. At first, the force-extension $f$ is known for values of $\Lambda_{e}^{\text {virgin }}$ corresponding to macroscopic stretches ranging in $1<\lambda_{e}<\lambda_{e \max }^{(i-1)}$, and therefore is used to compute $X\left(\Lambda_{\max }^{(i)}\right)$. In a second step, $X\left(\Lambda_{\max }\right)$ being defined for the entire cycle $(i), f$ is extended for values of $\Lambda_{e}^{\text {virgin }}$ corresponding to $\lambda_{e} \in\left[\lambda_{e \max }^{(i-1)}, \lambda_{e \max }^{(i)}\right]$. Computational details for both steps are provided in Tables 3 and 4 respectively. When both $X$ and $f$ have been determined for cycles up to $(i)$, the identification strategy is iterated for cycle $(i+1)$. Let us note that intermediate values for $f$ and $X$ are given by a linear interpolation.
Table 2

Identification procedure initialization.

$\begin{array}{cl}\text { Experimental data } & \text { First cycle maximum stretch } \lambda_{\max }^{(i=1)} \\ & \text { Couples }\left(\sigma_{11}, \lambda_{e}\right) \\ \text { Initial conditions } & X(1)=1 \\ & f(1)=0 \\ \text { Mullins criterion } & \text { Compute the directional governing } \\ & \text { parameter } \Lambda_{\max }(\boldsymbol{u}) \\ & \Lambda_{\max }(\boldsymbol{u})=\sqrt{\boldsymbol{u} \cdot \boldsymbol{C}\left(\lambda_{\max }^{(i=1)}\right) \cdot \boldsymbol{u}} \\ & \operatorname{Relationship~between}\left(\sigma_{11}, \lambda_{e}\right) \text { and }(X, f) \\ & \sigma_{11}=\iint_{\mathcal{S}} g\left(u_{1}^{2} \lambda_{e}^{2}-u_{2}^{2} / \lambda_{e}\right) \mathrm{d} \mathcal{S} \\ & \text { with } g=f\left(\Lambda_{e}\right) / \Lambda_{e}-f(1) \text {, and } \\ & \log \left(\Lambda_{e}\right)=X \log \left(\Lambda_{e}^{\text {virgin }}\right) \\ & \text { Compute } X\left(\Lambda_{\max }\right) \text { for } \Lambda_{\max }=\lambda_{\max }^{(i=1)} \text { and } \\ & f\left(\Lambda_{e}^{\text {virgin }}\right) \text { for } \lambda_{e}=\lambda_{e \max }^{(i=1)} \text { with a local square } \\ & \text { minimization of } \sigma_{11} \\ & \text { Compute few intermediate values of } \\ & f\left(\Lambda_{e}^{\text {virgin }}\right) \text { for } \lambda_{e} \in\left[1, \lambda_{e}^{(i=1)}\right] \text { with a local } \\ & \text { square minimization of } \sigma_{11} \text { and the } \\ & \text { computed } X \text { value } \\ & \end{array}$

During the identification procedure described above, evolutions of $X$ and $f$ are defined by experimental data only. However, it was noticed that the following restriction,

$f\left(\Lambda_{e}^{\text {virgin }} \leqslant 1\right)=0$

was favorable for a good comparison between the model and the experimental data in terms of induced anisotropy. While proof of such a restriction will be discussed in the next section, it may be noticed that this restriction may be interpreted as if directions in compression do not sustain stress but only stretched directions do. Anyhow, accounting for Eq. (17) within the proposed framework does not lead to any adjustment in the constitutive equations or the identification procedure previously presented.

The proposed identification procedure was tested on material A. A first cycle is performed up to $\lambda_{\max }^{(i=1)}=1.1$, then for each cycle, the maximum stretch was increased with a

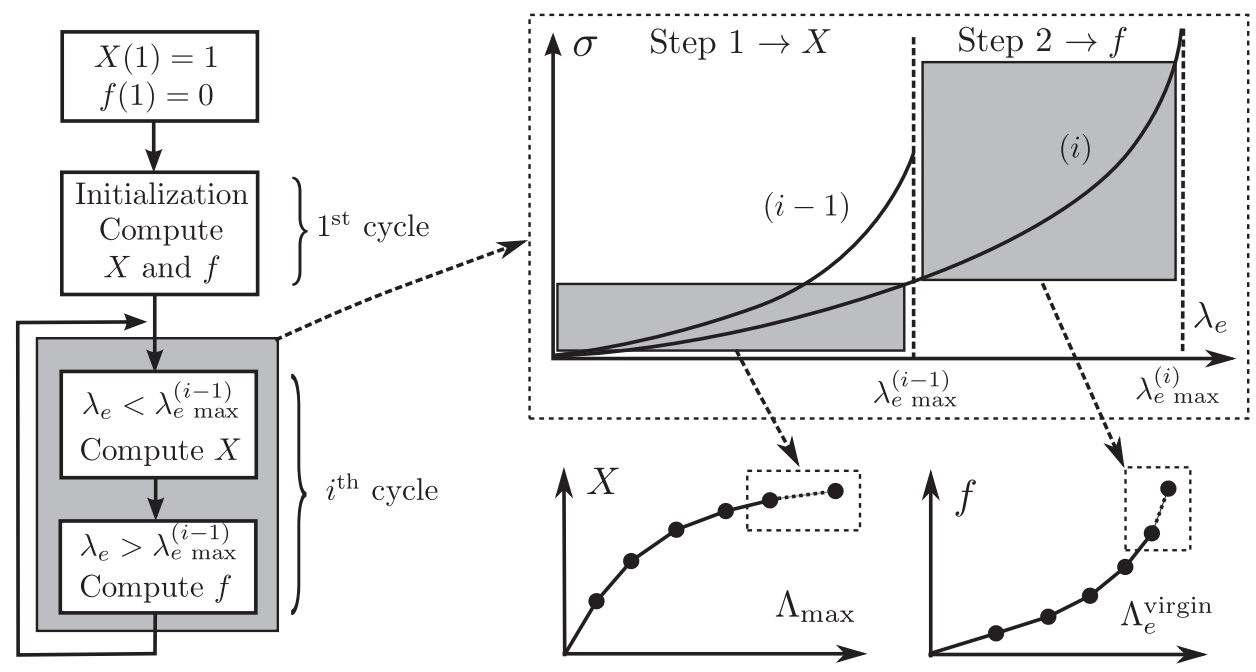

Fig. 6. Identification procedure. 
Table 3

First identification step at cycle $i$.

\begin{tabular}{cl}
\hline Experimental data & Cycle $i$ maximum stretch $\lambda_{\max }^{(i)}$ \\
& Couples $\left(\sigma_{11}, \lambda_{e}\right)$ for $\lambda_{e}<\lambda_{\max }^{(i-1)}$ \\
Initial conditions & $X\left(\Lambda_{\max }\right)$ known for $\Lambda_{\max } \in\left[1, \lambda_{\max }^{(i-1)}\right]$ \\
& $f\left(\Lambda_{e}^{\text {virgin }}\right)$ known for $\lambda_{e} \in\left[1, \lambda_{e \max }^{(i-1)}\right]$ \\
& Compute the directional governing \\
Mullins criterion & parameter $\Lambda_{\max }(\boldsymbol{u})$ \\
& $\Lambda_{\max }(\boldsymbol{u})=\sqrt{\boldsymbol{u} \cdot \boldsymbol{C}\left(\lambda_{\max }^{(i)}\right) \cdot \boldsymbol{u}}$ \\
& $\operatorname{Relationship~between~}\left(\sigma_{11}, \lambda_{e}\right)$ and $(X, f)$ \\
& $\sigma_{11}=\iint_{\mathcal{S}} g\left(u_{1}^{2} \lambda_{e}^{2}-u_{2}^{2} / \lambda_{e}\right) \mathrm{d} \mathcal{S}$ \\
& with $g=f\left(\Lambda_{e}\right) / \Lambda_{e}-f(1)$, and \\
& $\log \left(\Lambda_{e}\right)=X \log \left(\Lambda_{e}^{\text {virgin }}\right)$ \\
& Compute $X\left(\Lambda_{\max }\right)$ for $\Lambda_{\max }=\lambda_{\max }^{(i)}$ with a \\
& mean square minimization of $\sigma_{11}$ on the \\
& interval $\Lambda_{\max } \in\left[\lambda_{\max }^{(i-1)}, \lambda_{\max }^{(i)}\right]$ \\
\hline
\end{tabular}

Table 4

Second identification step at cycle $i$.

\begin{tabular}{ll}
\hline Experimental data & Couples $\left(\sigma_{11}, \lambda_{e}\right)$ for $\lambda_{e}>\lambda_{\max }^{(i-1)}$ \\
Initial conditions & $X\left(\Lambda_{\max }\right)$ known for $\Lambda_{\max } \in\left[1, \lambda_{\max }^{(i)}\right]$ \\
& $f\left(\Lambda_{e}^{\text {virgin }}\right)$ known for $\lambda_{e} \in\left[1, \lambda_{e \max }^{(i-1)}\right]$ \\
& Relationship between $\left(\sigma_{11}, \lambda_{e}\right)$ and $(X, f)$ \\
& using \\
& $\sigma_{11}=\iint_{\mathcal{S}} g\left(u_{1}^{2} \lambda_{e}^{2}-u_{2}^{2} / \lambda_{e}\right) \mathrm{d} \mathcal{S}$ \\
& with $g=f\left(\Lambda_{e}\right) / \Lambda_{e}-f(1)$, and \\
& $\log \left(\Lambda_{e}\right)=X \log \left(\Lambda_{e}^{\text {virgin }}\right)$ \\
& $\operatorname{Compute}$ few values of $f\left(\Lambda_{e}^{\text {virgin }}\right)$ for \\
& $\lambda_{e} \in\left[\lambda_{e}^{(i-1)}, \lambda_{e m a x}^{(i)}\right]$ with a local square \\
& minimization of $\sigma_{11}$ \\
\hline
\end{tabular}

step of $\Delta \log (\lambda)=0.1$. The interest of such a loading stands in smaller first few cycles before the difference between two successive cycles becomes significant. Therefore, the loading is well suited for the identification, with a short cycle for initialization (Table 2) and stretch intervals increasing at each cycle for the computation of $f$ (Table 4). Model fit of the experimental unloading responses used for identification is shown in Fig. 7. Experimental responses appear to be successfully represented.

Fig. 8a shows the elementary force-extension relation, $f\left(\Lambda_{e}^{\text {virgin }}\right)$, resulting from the identification procedure. One may notice that $f$ presents the classic features of a filled rubber behavior, with a low stiffness and a quasi-linear response at small stretch, then a sharp upturn followed by an asymptotic vertical at larger stretch when the material limit extensibility is reached.

The Mullins softening rule, $X\left(\Lambda_{\max }\right)$, is shown in Fig. $8 \mathrm{~b}$. Considering the maximal stretch undergone by the material before failure, the dependence of $X$ to the maximum extension is well approximated by,

$X=\alpha \log \left(\Lambda_{\max }\right)$

with $\alpha$ a material parameter characterizing the softening evolution rate. For material A, one gets $\alpha=8.8$. The logarithmic evolution of $X$ with respect to the maximum extension is consistent with previous results aiming at

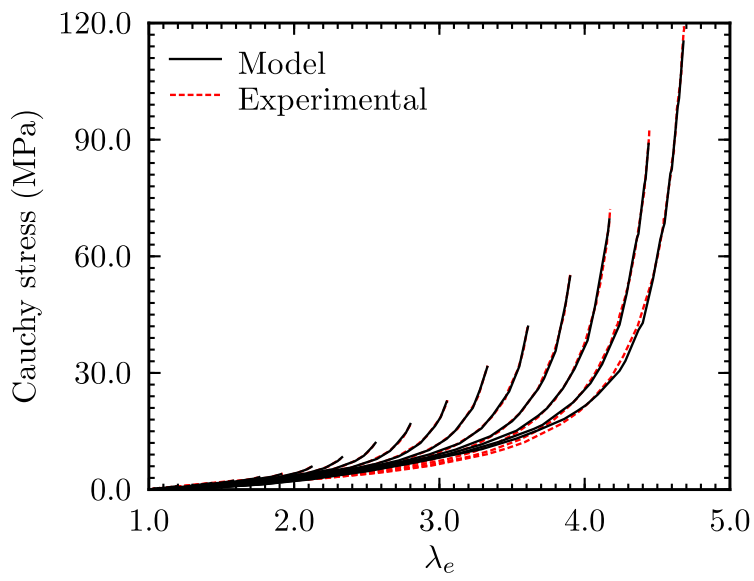

Fig. 7. Model ability to fit the Mullins softened behavior of material A.

characterizing the Mullins softening (Merckel et al., 2011). Furthermore, softening evolution rules defined in order to converge toward a saturation limit are used in other models, for instance Miehe and Keck (2000), Klüppel and Schramm (2000) and Qi and Boyce (2004) among others.

\section{Results and discussion}

\subsection{Prediction of non-proportional loading resulting behavior}

This section aims at illustrating the model predictive capabilities. For this purpose, the force-extension relation, $f\left(\Lambda_{e}^{\text {virgin }}\right)$, and the Mullins softening law, $X\left(\Lambda_{\max }\right)$, previously identified for the material $A$ are used to represent the responses of material A when submitted to cyclic uniaxial tension post non-proportional pre-stretchings.

Samples submitted to cyclic uniaxial tension tests are now small dumbbell samples punched in larger samples already submitted to a uniaxial or biaxial pre-loading. Between both the pre-loading and the loading experiments, an important residual stretch recovery induced by the material viscoelasticity occurs. The material viscoelasticity is not accounted for here and the experimental data are corrected according to Eq. (15) and the modeling condition $\boldsymbol{F}_{p}=\boldsymbol{I}$ is set.

First, the experimental procedure is applied for $\lambda=2.5$ uniaxial stretch pre-loading, and small dumbbell samples are cut at $45^{\circ}$ and $90^{\circ}$ from the pre-loading stretching direction. The cyclic uniaxial tension is performed with maximum stretches increasing of $\Delta \lambda=0.25$ at each cycle. According to criterion Eq. (11), the Mullins softening is activated in some directions from the very first cycle, and it evolves differently according to the directions. Comparison between the experimental unloading responses and the model predictions are shown in Fig. 9. One may notice that the experimental unloading curves are well approximated for small and large cycles and this without using any adjusting parameter or further identification but by using the material functions $f$ and $X$ identified earlier on a virgin sample only (Section 3.3 ). Therefore, the model is 
(a)

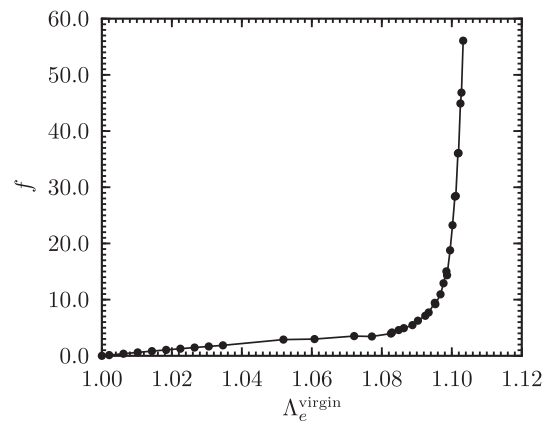

(b)

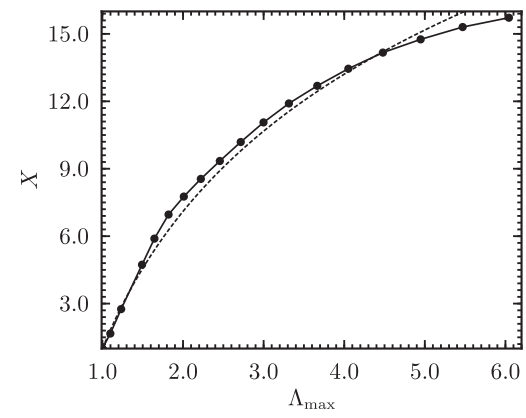

Fig. 8. Identification results for material A. (a) Elementary force-extension relation. (b) Mullins softening rule.

(a)

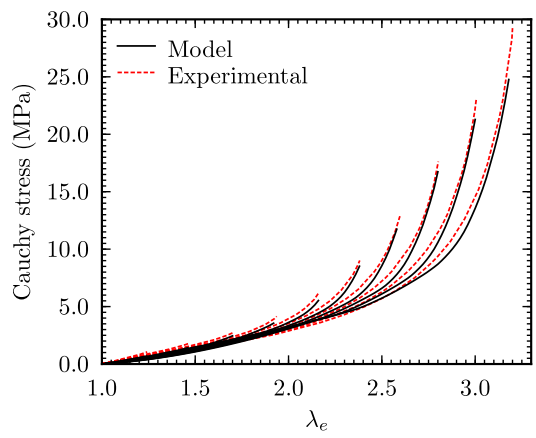

(b)

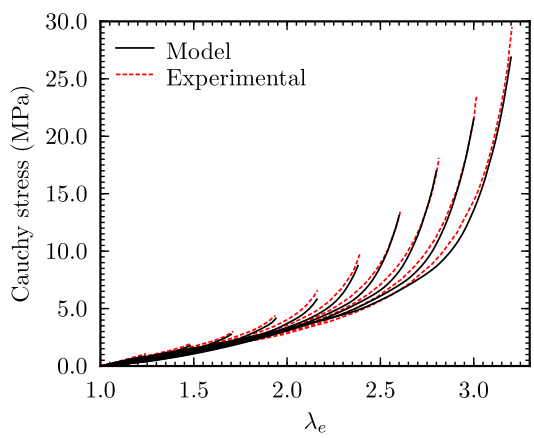

Fig. 9. Model prediction for 2.5-uniaxially pre-stretched material. Sample cut in directions (a) $45^{\circ}$ and (b) $90^{\circ}$ compared to direction of pre-stretching.

(a)

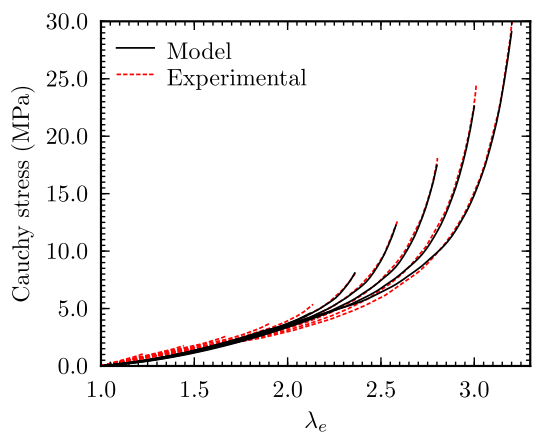

(b)

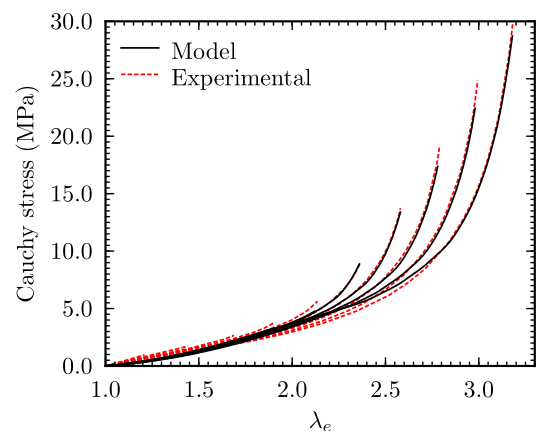

Fig. 10. Model prediction of the uniaxial stress-stretch responses of samples biaxially pre-stretched material up to (a) $F_{11}=F_{22}=2.5$ and (b) $F_{11}=2.5, F_{22}=1.75$ and cut along the maximum pre-stretched direction.

able to capture the material anisotropy induced by the Mullins softening resulting from a uniaxial pre-loading. Let us note that such a Mullins softening observed at the very first cycles in Fig. 9a and b, could not be represented with an isotropic Mullins criterion, which would have predicted Mullins reactivation at $\lambda=2.5$ only.

Second, the same experimental procedure is applied to samples submitted to biaxial pre-loads. Two pre-loading conditions were chosen, $F_{11}=F_{22}=2.5$ equi-biaxial pre-stretching and $F_{11}=2.5$ and $F_{22}=1.75$ biaxial prestretching. The small dumbbell samples were cut in the direction of maximum stretching (direction 1). According to criterion Eq. (11), the Mullins softening should not activate until $\lambda=2.5$ was reached. Experimental responses and model estimates are compared in Fig. 10. Apart from the viscoelasticity exhibited by actual samples at the very beginning of the unloadings that cannot be reproduced, the model predictions appear to be accurate below and above 
(a)

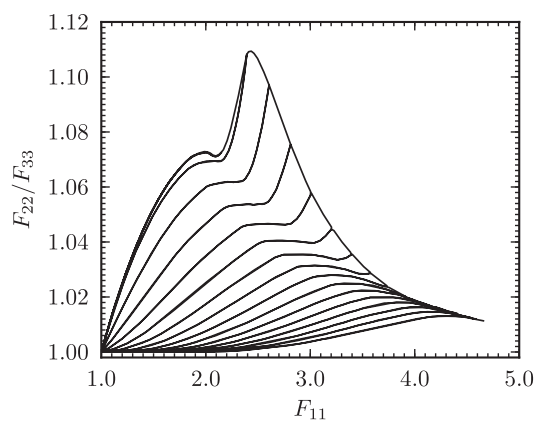

(b)

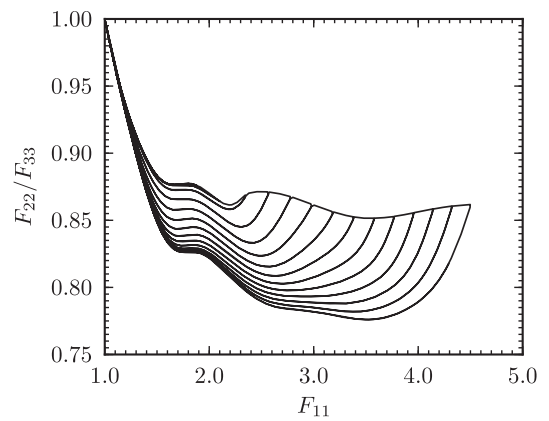

Fig. 11. Model prediction for the anisotropy changes in-plane (2,3). (a) With Eq. (17) condition. (b) Without Eq. (17) condition.

(a)

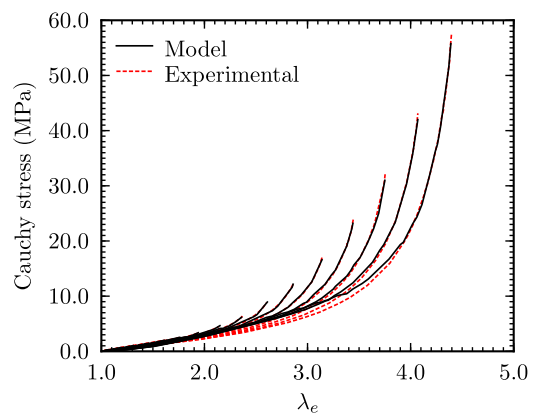

(c)

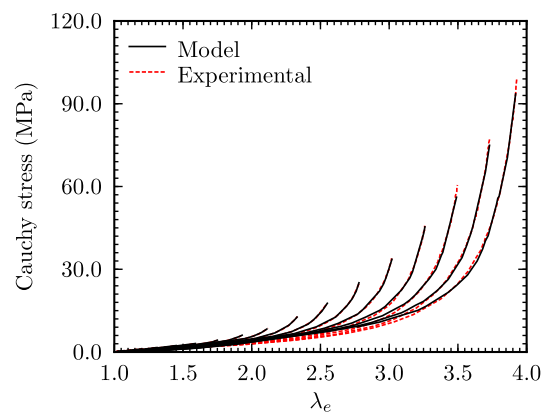

(b)

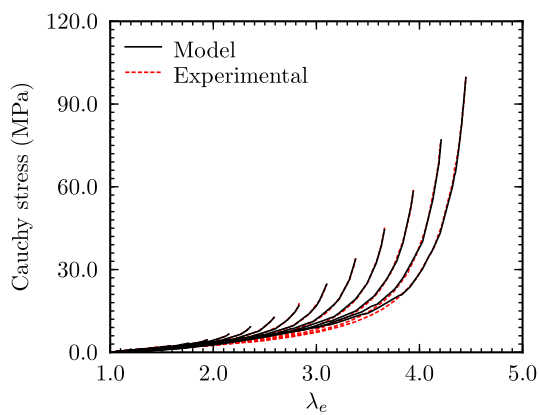

(d)

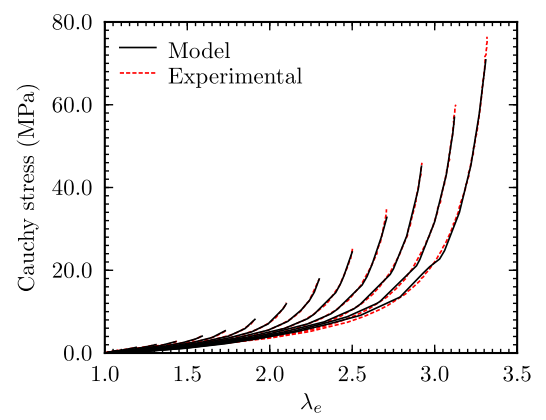

Fig. 12. Model prediction for material (a) B1, (b) B2, (c) B3, (d) B4.

the Mullins activation for both pre-loading conditions. The softening induced by a biaxial loading appears to be also well captured by the model.

Results shown in Fig. 10 yield to important consequences in terms of material behavior modeling and identification. The mechanical tests performed in order to obtain the experimental data involved a multiaxial loading path, but the Mullins softening evolution rule $X\left(\Lambda_{\max }\right)$ was chosen as dependent of the maximal directional extension only. The prediction abilities shown by the model proves that $X$ does not require complex account of the loading multiaxiality and its complete identification may be performed on a mere cyclic uniaxial tension test.

The following section aims at studying the actual material anisotropy evolution and its model prediction. The anisotropy is then characterized by measuring and comparing the stretches according to the principal stretching directions.

\subsection{Estimate of the induced anisotropy}

The ratio $F_{22} / F_{33}$ evolution was introduced Section 2.4 to characterize the anisotropy changes during cyclic uniaxial tests. Fig. 11a shows the ratio $F_{22} / F_{33}$ model prediction for a cyclic uniaxial loading performed on a 2.5-equi-biaxially pre-stretched material. As long as $\lambda=2.5$ is not reached, the Mullins softening does not evolve and $F_{22} / F_{33}$ path remains identical. Once the Mullins softening is re-activated, changes occurs and at each cycle, the path slowly converges on a proportional uniaxial loading path 
(a)

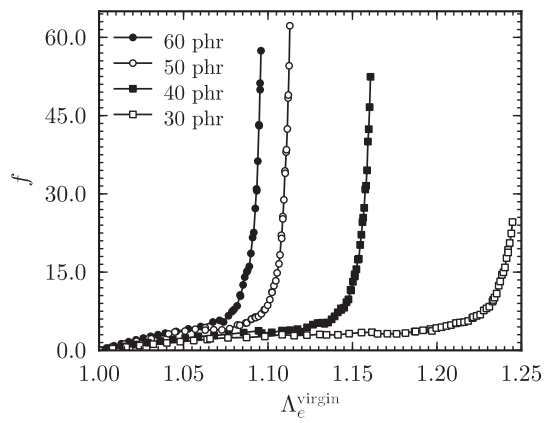

(b)

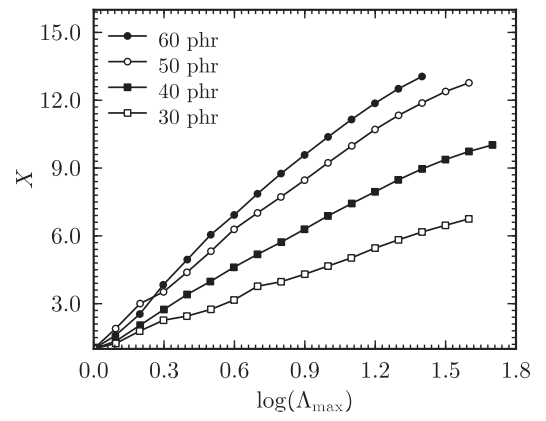

Fig. 13. Identification results for material with different amount of fillers. (a) Elementary force-extension functions. (b) Mullins softening rules.

(i.e. $F_{22} / F_{33}=1$ ). This modeling result is to be compared with experimental observations presented Fig. 3b. Note that the discrepancies between Figs. 3a and 11 are due to the model initial isotropy assumption, which does not match the actual material initial anisotropy. Nonetheless, the trend of the material anisotropy evolution is well captured by the model.

In the modeling section, the condition Eq. (17) was introduced for the elementary force-extension. When releasing this condition, the fitting procedure drives to a function $f$ reaching negative values for $\lambda<1$. The resulting ratio $F_{22} / F_{33}$ computed with the function $f$ obtained without applying condition Eq. (17) is shown in Fig. 11b. During the equi-biaxial pre-loading, the material is softened in direction 2 while remaining virgin in direction 3. Since the material stiffness is higher in direction 3 , boundary conditions $\sigma_{22}=\sigma_{33}=0$ should yield to $F_{22} / F_{33}>1$. This is obviously not the case in Fig. 11b. Moreover, one may notice in Fig. 11b that the anisotropy intensity increases with $\lambda_{\max }$ while it is expected to decrease. The result is obviously unrealistic and validate the condition Eq. (17), which supports the physical picture of directions in compression not sustaining the stresses.

\subsection{Other model interest: Study of the effect of filler amount}

Hitherto, only the reference material labeled A in Table 1 was used to develop the mechanical behavior model and the identification procedure. This section aims at applying the model on various materials in order to validate the identification procedure and to investigate the effect of the filler amount. Materials B1, B2, B3 and B4 described in Table 1 were submitted to cyclic uniaxial loadings with a maximum stretch increasing at each cycle of $\Delta \log (\lambda)=0.1$ step and up to failure. The evolution rules $f$ and $X$ were computed for each material performing the identification procedure detailed in Section 3.3. The model responses and experimental data are favorably compared in Fig. 12. Therefore, the model and the identification procedure was successfully extended to several materials, exhibiting distinct mechanical behaviors and distinct sensitivities to the Mullins softening.

In order to investigate dependencies to the filler amount, evolution rules $f$ and $X$ are compared in Fig. 13 .
Fig. 13a shows the filler amount effect on the identified elementary force-extension relations. Every material exhibits a quasi-linear virgin response followed by a plateau ended by a sharp upturn. The main dependences of $f$ to the filler amount are the initial stiffness increase and the upturn stretch decrease with the filler amount. These observations are consistent with former results from the literature. Actually, the reinforcing effect of filler volume fraction on the initial stiffness is well known (Einstein, 1906; Guth and Gold, 1938).

The effect of the amount of fillers on $X$ is illustrated Fig. 13b. As expected, the Mullins softening rate increases with the amount of fillers, see for instance Mullins and Tobin (1957), Bergström and Boyce (1999), Klüppel and Schramm (2000), Dorfmann and Ogden (2004) and Merckel et al. (2011) among others. One may notice that for every material the evolution of $X$ is quasi-linear with respect to the logarithm of the maximum directional stretch $\Lambda_{\max }$. The same property was observed and shown in Fig. 8b for material A, therefore the Mullins softening evolution rule introduced in Eq. (18) may well be general.

\section{Conclusion}

This contribution aimed at proposing a constitutive model for the mechanical behavior of filled rubbers with Mullins softening. The constitutive equations were grounded on an thorough analysis of original experimental data. Basic uniaxial tensile tests and unconventional nonproportional tensile tests including uniaxial and biaxial loading paths were used to produce the necessary experimental data. The model was based on a directional approach in order to capture the anisotropy induced by general non-proportional pre-loading histories. The Mullins softening was accounted for by the strain amplification concept and was activated by a directional criterion. The framework was developed in order to avoid any a priori assumption of the mathematical forms of the elementary strain energy density and of the Mullins softening evolution rule. An original identification procedure was proposed in order to build both functions from a cyclic tensile stress-stretch response. An accurate fit of the experimental data provided by a cyclic proportional uniaxial tensile test illustrated the model ability to capture the 
material stress-softening. Once identified on a proportional cyclic test, the model was shown to successfully and accurately predict uniaxial stress-stretch responses for nonproportional uniaxially or biaxially pre-stretched samples. Finally, the model and the identification procedure were applied on various filled rubber materials evidencing different mechanical behaviors and sensitivities to the Mullins softening. The results showed favorable comparisons and illustrated the model flexibility to apply to a wide range of rubber-like materials.

\section{Acknowledgments}

This work was supported by the French "Agence Nationale de la Recherche" through project AMUFISE (MATETPRO 08-320101). The authors acknowledge useful discussions with D.Berghezan, C. Creton, J. de Crevoisier, F. Hild, C. Moriceau, M. Portigliatti, S. Roux, F. Vion-Loisel, and $\mathrm{H}$. Zhang.

\section{References}

Bazănt, Z., Oh, B., 1986. Efficient numerical integration on the surface of a sphere. Z. Angew. Math. Mech. 66, 37-49.

Bergström, J.S., Boyce, M.C., 1999. Mechanical behavior of particle filled elastomers. Rubber Chem. Technol. 72, 633-656.

Bouasse, H., Carrière, Z., 1903. Sur les courbes de traction du caoutchouc vulcanisé. Ann. Fac. Sci. Toulouse 3, 257-283.

Dargazany, R., Itskov, M., 2009. A network evolution model for the anisotropic Mullins effect in carbon black filled rubbers. Int. J. Solids Struct. 46 (16), 2967-2977.

Diani, J., Brieu, M., Vacherand, M., 2006. A damage directional constitutive model for Mullins effect with permanent set and induced anisotropy. Eur. J. Mech. A Solids 25, 483-496.

Diani, J., Brieu, M., Vacherand, M., Rezgui, A., 2004. Directional model for isotropic and anisotropic hyperelastic rubber-like materials. Mech. Mater. 36, 313-321.

Dorfmann, A., Ogden, R., 2004. A constitutive model for the mullins effect with permanent set in particle-reinforced rubber. Int. J. Solids Struct. 41, 1855-1878.

Einstein, A., 1906. Eine neue bestimmung der molekuldimensionen. Ann. Phys. (Leipzig) 19, 289.

Göktepe, S., Miehe, C., 2005. A micro-macro approach to rubber-like materials. Part III: The micro-sphere model of anisotropic Mullinstype damage. J. Mech. Phys. Solids 53 (10), 2259-2283.

Guth, E., Gold, O., 1938. On the hydrodynamical theory of the viscosity of suspensions. Phys. Rev. 53, 322.

Hanson, D.E., Hawley, M., Houlton, R., Chitanvis, K., Rae, P., Orler, B.E., Wrobleski, D.A., 2005. Stress softening experiments in silica-filled polydimethylsiloxane provide insight into a mechanism for the Mullins effect. Polymer 46 (24), 10989-10995.

Itskov, M., Halberstroh, E., Ehret, A.E., Vöhringer, M.C., 2006. Experimental observation of the deformation induced anisotropy of the mullins effect in rubber. Kaut. Gummi. Kunstst., 93-96.
Klüppel, M., Schramm, J., 2000. A generalized tube model of rubber elasticity and stress softening of filler reinforced elastomer systems. Macromol. Theory. Simul. 9, 742-754.

Laraba-Abbes, F., Ienny, P., Piques, R., 2003. A new Tailor-made methodology for the mechanical behaviour analysis of rubber-like materials. Part II: Application to the hyperelastic behaviour characterization of a carbon-black filled natural rubber vulcanizate. Polymer 44 (3), 821-840.

Le Cam, J., 2010. A review of the volume changes in rubbers: the effect of stretching. Rubber Chem. Technol. 83 (3), 247-269.

Machado, G., 2011. Contribution à l'étude de l'anisotropie induite par l'effet mullins dans les élastomères silicone chargés. Ph.D. thesis, Université de Grenoble.

Merckel, Y., Brieu, M., Diani, J., Caillard, J., 2012. A Mullins softening criterion for general loading conditions. J. Mech. Phys. Solids 60, 1257-1264.

Merckel, Y., Diani, J., Brieu, M., Caillard, J., 2011. Characterization of the Mullins effect of carbon-black filled rubbers. Rubber Chem. Technol. 84 (3).

Miehe, C., Keck, J., 2000. Superimposed finite elastic-viscoelasticplastoelastic stress response with damage in filled rubbery polymers. Experiments, modelling and algorithmic implementation. J. Mech. Phys. Solids 48, 323-365.

Mullins, L., 1947. Effect of stretching on the properties of rubber. J. Rubber Res. 16 (12), 275-289.

Mullins, L., 1949. Permanent set in vulcanized rubber. India Rubber World $120,63-66$.

Mullins, L., 1950. Thixotropic behavior of carbon black in rubber. J. Phys. Col. Chem. 54 (2), 239-251.

Mullins, L., 1969. Softening of rubber by deformation. Rubber Chem. Technol. 42, 339-362.

Mullins, L., Tobin, N., 1957. Theoretical model for the elastic behavior of filler-reinforced vulcanized rubber. Rubber Chem. Technol. 30, 555571.

Mullins, L., Tobin, N., 1958. Carbon-black loaded rubber vulcanizates: volume changes in stretching. Rubber Chem. Technol. 31, 505-512.

Pawelski, H., 2001. Softening behaviour of elastomeric media after loading in changing directions. In: Besdo, D., Schuster, R.H., Ihleman, J. (Eds.), Proceedings of the Second European Conference of the Constitutive Models for Rubber. Balkema, pp. 27-34.

Qi, H., Boyce, M., 2004. Constitutive model for stretch-induced softening of the stress stretch behavior of elastomeric materials. J. Mech. Phys. Solids 52, 2187-2205.

Shinomura, T., Takahashi, M., 1970. Volume change measurements of filled rubber vulcanizates under stretching. Rubber Chem. Technol. 43, 1025-1035.

Treloar, L., Riding, G., 1979. A non-gaussian theory for rubber in biaxial strain. Part I. Mechanical properties. In: Proc. Roy. Soc. Lond. 369 (ser. A), pp. 261-280.

Weiss, A., Maker, B., Govindjee, S., 1979. Finite element implementation of incompressible, transversely isotropic hyperelasiticity. Comput. Methods Appl. Mech. Eng. 135, 107-128.

Zhang, H., Scholz, A.K., de Crevoisier, J., Vion-Loisel, F., Besnard, G., Hexemer, A., Brown, H.R., Kramer, E.J., Creton, C., 2012. Nanocavitation in carbon black filled styrene-butadiene rubber under tensiondetected by real time small angle X-ray scattering. Macromolecules 45, 1529-1543. 